\title{
Pénétration statique dans les graviers sableux denses, la molasse et les marnes compactes
}

\section{G. SANGLERAT}

Expert honoraire agréé par la Cour de Cassation

Lyon

M. PETIT-MAIRE

PDG d'AMAP'sols

Saint-Héand (Loire)

F. BARDOT

Expert agréé

par la Cour de Cassation

Lyon

P. SAVASTA

Président de SETSOL

Velaux (Bouches-du-Rhône)
- U. Une légende tenace voudrait faire croire que la pénétration statique ne peut être utilisée ni dans les graviers, ni dans la molasse, ni dans les marnes compactes.

Les auteurs décrivent un nouveau pénétromètre statique. qui permet de traverser de tels sols. Ils donnent des exemples où la résistance de pénétration statique $q_{\text {, }}$ dépasse $85 \mathrm{MPa}$ dans des marnes et peut atteindre $148 \mathrm{MPa}$ dans les graviers compacts ou la molasse dense.

\section{Static penetration in dense gravel, sandstone and hard claystone}

There is a long-established belief that supposes static

penetration cannot be used in gravels, sandstones, claystones or soft rock.

The authors describe a new static penetrometer which does penetrate these types of soils. They give examples where the static cone resistance, $q_{\text {, }}$ exceeds $85 \mathrm{MPa}$ in claystone and capable of reaching $148 \mathrm{MPa}$ in dense gravels or in sandstone. 


\section{Introduction}

Depuis des années, il était communément admis que la pénétration statique n'était pas possible pour reconnaitre ni les couches de graviers, ni les couches de marne, ni la molasse.

Cette opinion provenait de ce que, avec la plupart des pénétromètres statiques, ces couches, rencontrées même à faible profondeur, bloquaient l'appareil et provoquaient, par ailleurs, des détériorations graves dans les cônes électriques, ce qui était très coûteux.

De ce fait, certains géotechniciens ont limité l'utilisation du pénétromètre statique aux couches molles, alors que d'autres acceptaient son utilisation dans les sables (Begemann, 1965: Schmertmann, 1967) mais, en général tous l'excluaient pour l'étude des graviers (Mitchell, 1998).

Cependant, cette restriction n'a plus de raison d'être depuis 1992 (Sanglerat, 1994; Sanglerat et al., 1995).

Les exemples caractéristiques ci-dessous, obtenus avec le pénétromètre AMAP'sols ouvrent de nouvelles perspectives à la pénétration statique.

Le nom AMAP'sols est une contraction de Ateliers Mobiles d'Auscultation par Pénétration des Sols.

\section{2}

\section{Le pénétromètre statique-dynamique AMAP'sols}

\section{1}

\section{Principe}

L'histoire de la pénétration statique-dynamique a èté présentée par Sanglerat en 1979 [6].

En 1992, des géotechniciens de Lyon et de SaintÉtienne décidèrent de créer un nouveau pénétromètre statique-dynamique, afin de perfectionner ce type de reconnaissance des sols.

Les améliorations très importantes apportées peuvent ètre résumées comme suit :

\section{En statique :}

Fonctionnement entièrement automatique. Enfoncement à une vitesse rigoureusement constante de $2 \mathrm{~cm} /$ seconde, avec enregistrement numérique en continu des mesures sur carte mémoire, avec sortie simultanée des diagrammes des efforts tracés en temps réel permettant le contróle instantané des opérations.

Les données enregistrées sont transmises par modem, ce qui permet de tracer très rapidement les courbes représentatives des essais dans le bureau de l'Ingénieur chargé du projet et d'extraire, si besoin est, toutes les valeurs numériques nécessaires aux calculs de force portante ou de tassements [Mitchell et al., 1998; Sanglerat et al, 1984; Lunne et al., 1998].

\section{En dynamique :}

Le battage par chute d'un mouton a été remplacé par un très puissant marteau hydraulique à frappes rapides et réglables, par série de coups rapprochés, permettant de traverser des couches extrèmement dures ou de pénétrer le substratum rocheux altéré.

\section{Installation :}

L'appareil est monté sur un camion Mercedes $6 \times 6$ de 250 kN (Fig. 1).

Les réglages de mise à niveau de l'appareil, pour enfoncer verticalement les tiges, s'effectuent très rapidement, grãce à cing vérins hydrauliques commandés par des électrodistributeurs contrôlés électriquement. Quatre de ces vérins sont ceux appartenant au dispositif du train de chenilles auxiliaire dont est muni le véhicule porteur (Fig, 2); ce dispositif lui confère des possibilités d'accès et d'évolution sur des sols très mous où tous les autres véhicules s'enlisent, ainsi que sur des terrains en forte pente.

Le système de pénétration statique-dynamique a été conçu et construit à Lyon (Fig. 3) et monté en Hollande, sur un camion Mercedes, par Van den Berg, grand spécialiste du pénétromètre statique depuis de lonques années, qui a apporté sa technicité et notamment son expérience dans l'ensemble des applications de l'hydraulique et de l'acquisition numérique des données.
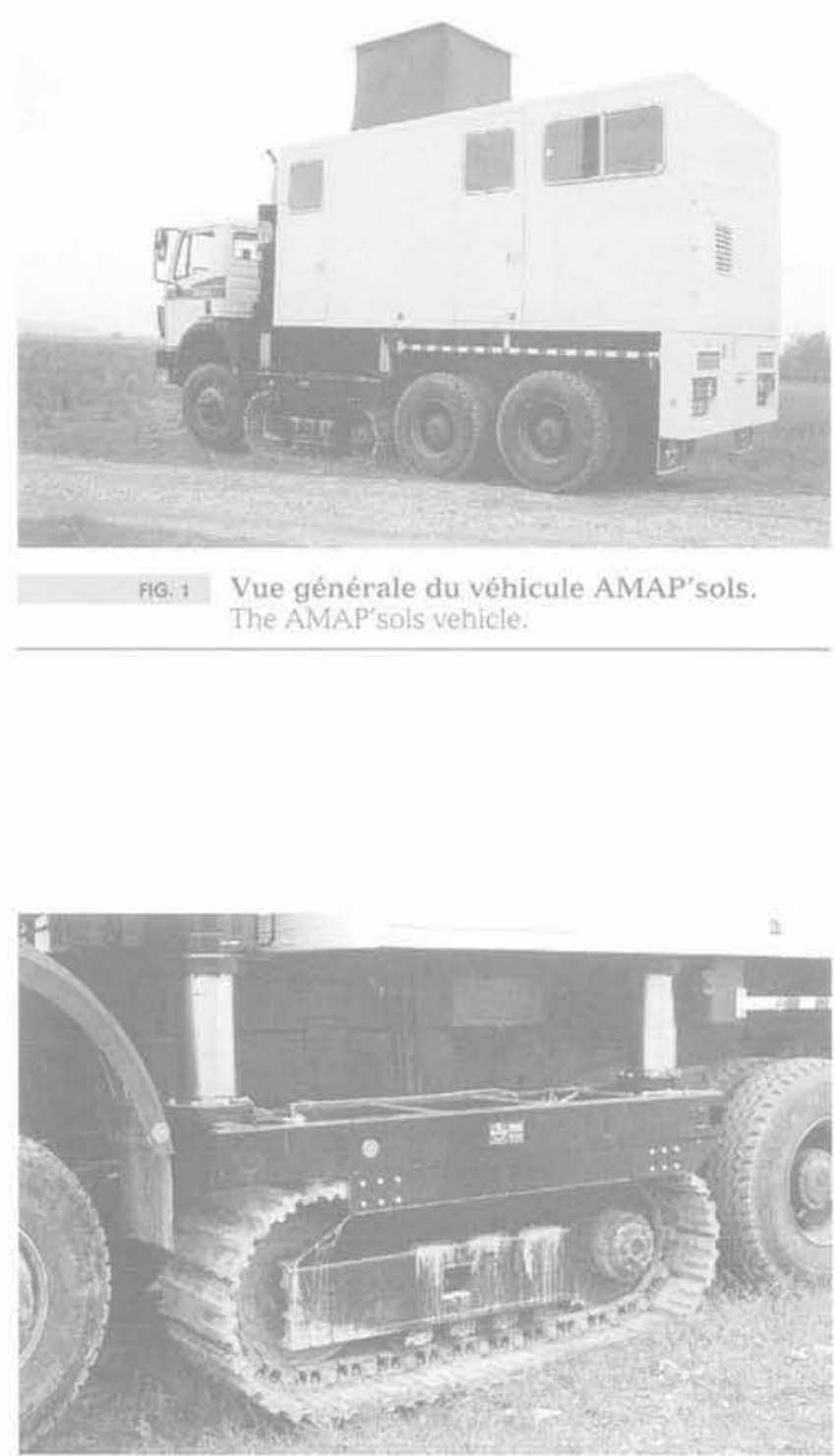

FIG. 2 Vérins de stabilisation sur train de chenilles.

Hydraulic stabilisers on caterpillar track. 


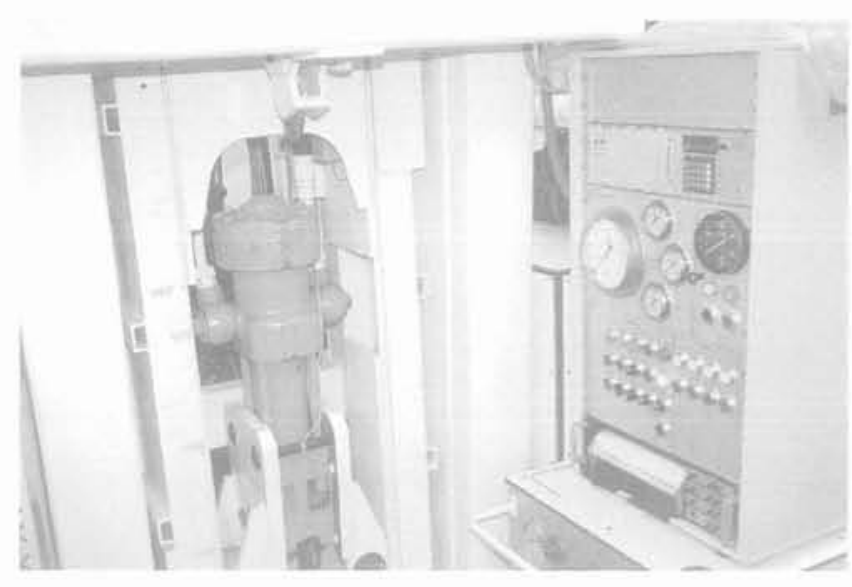

FG. 3 Vue du marteau hydraulique et des appareils de mesures et de contrôles de pénétration statique-dynamique AMAP'sols.

View of the hydraulic hammer and measuring equipment for the static-dynamic penetration of AMAP'sols.

\section{Caractéristiques}

Ce pénétromètre permet de réaliser tous les sys. tèmes de pénétration dans le sol, ainsi que des essais spéciaux associés à la pénétration. II peut utiliser tous les cônes de pénétration connus actuellement, de $10 \mathrm{~cm}^{2}$ à $50 \mathrm{~cm}^{2}$, à transmission mécanique ou à acquisition électrique, ainsi que les piézocônes.

En pénétration statique, on utilise d'une manière courante soit une pointe de $80 \mathrm{~mm}$ de diamètre (còne dit de $50 \mathrm{~cm}^{2}$ ) avec un manchon de frottement latéral de $250 \mathrm{~mm}$ de long (Fig. 4), soit une pointe de $75 \mathrm{~mm}$ (cône dit de $44 \mathrm{~cm}^{2}$ ) avec manchon de $200 \mathrm{~mm}$.

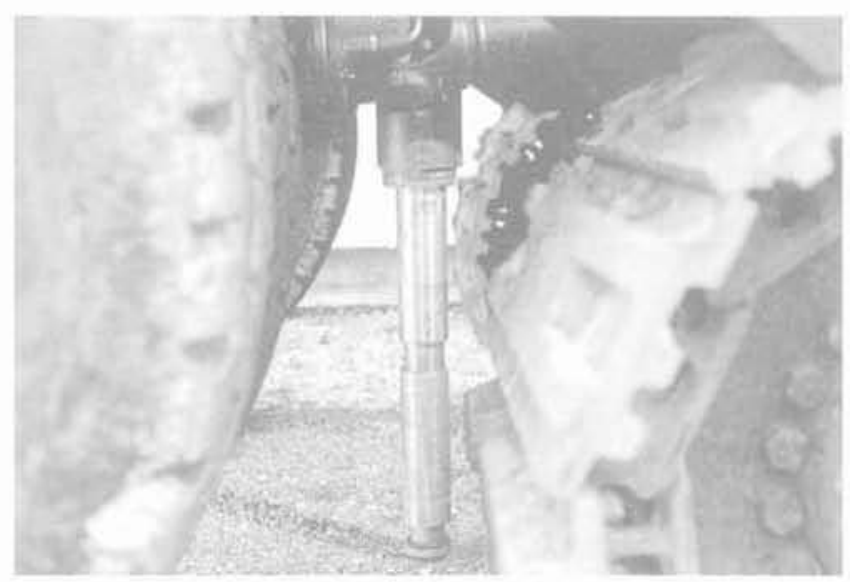

Fig. 4 Cône AMAP'sols de $50 \mathrm{~cm}^{2}$ avec manchon de frottement latéral.

AMAP'sols $50 \mathrm{~cm}^{2}$ cone with lateral friction sleeve.

Naturellement, on mesure en continu :

- q résistance de pointe (jusqu'à $30 \mathrm{MPa}$ ) ;

- f frottement latéral unitaire sur manchon ce qui permet de calculer le friction ratio FR:

- $\mathrm{Q}_{\mathrm{sr}}$ effort total d'enfoncement (jusqu'à $220 \mathrm{kN}$ mais limité généralement à $180 \mathrm{kN}$ ).

Les mesures sont enregistrées tous les $2 \mathrm{~cm}$.

Lorsque les cônes de $44 \mathrm{~cm}^{2}$ ou $50 \mathrm{~cm}^{2}$, avec manchon de frottement, sont arrêtés par une couche dure, on poursuit la pénétration statique avec une pointe plus petite, d'un diamètre de $39 \mathrm{~mm}$, dite de $12 \mathrm{~cm}^{2}$ (sans manchon de frottement), qui permet d'obtenir des valeurs de résistance de pointe très élevées pouvant atteindre jusqu'à $148 \mathrm{MPa}$ (on appelle souvent cette résistance de pointe $q_{122}$ pour la différencier de $\mathrm{q}_{c 50}$ ou de $\left.\mathrm{q}_{\mathrm{ct}+4}\right)$; au-delà, on passe en dynamique.

Lors de la pénétration dynamique, à titre d'information. on procède tous les $25 \mathrm{~cm}$ à une mesure de contróle en statique de l"effort total (pointe et frottement latéral) que l'on limite volontairement à $180 \mathrm{kN}$. Cela correspond, dans les milleux pulvérulents, à une résistance statique de pointe $q_{c i p}$ généralement de l'ordre de 130 à $140 \mathrm{MPa}$ (ce qui est considérable et n'est atteint par aucun autre pénétromètre).

Naturellement, chaque fois que I on constate que l'effort total statique Q. est inférieur à 160 ou $170 \mathrm{kN}$, on reprend la pénétration en statique puisqu'alors q est inférieur à 130 ou $140 \mathrm{MPa}$.

En cas de chute brutale de la résistance, le dispositif de battage s'arréte automatiquement et instantanément au toit de la couche moins résistante. Cette dernière est alors reconnue en pénétration statique sur toute son épaisseur, ce qui n'était pas toujours possible avec les anciens pénétromètres statiques-dynamiques qui souvent poinconnaient brutalement les premiers décimètres de ces couches de moindre résistance.

Un dispositif d'alerte sonore permet d'éviter de dépasser les charges maximales prévues pour les différentes tiges assurant la transmission des efforts jusqu'aux capteurs de mesures.

\section{3}

\section{Utilisations complémentaires}

Il est possible dutiliser sur le même apparell d'autres cônes, en particulier le piézocône et lenvirocône Van den Berq $[8,9]$. Naturellement, dans ces deux cas, on utilise des logiciels spécifiques élaborés par Van den Berg.

Le piézocône permet, selon l'usage, de déterminer $\mathrm{q}_{\mathrm{c}}, \mathrm{f}$, et la pression interstitielle $u$.

Avec l'envirocône, on mesure, outre q, les caractéristiques suivantes :

- conductivité :

- teneurs en $\mathrm{O}^{-+}$et $\mathrm{H}^{\text {: }}$

- potentiel Redox:

- températures :

$-\mathrm{pH}$ ?

- pression interstitielle.

L'ensemble de ces possibilités constitue une amélioration considérable des movens de reconnaissance et permet de traiter de nombreux problèmes d'environnement ou de waste management crâce à la qualité et à la fiabilité des renseignements recueillis $[3,9,10]$.

On peut par ailleurs utiliser un còne sismique.

On peut également, dans les sols cohérents, prélever des échantillons intacts sous gaine PVC de gros diamètre $(\varnothing 70 \mathrm{~mm})$.

Ce nouveau pénétromètre statique-dynamique a déjà fait l'objet de deux communications lors de conférences internationales $[4,5]$.

Diffèrents cas d'utilisation sont rapportés ci-dessous. 


\section{Pénétration dans la molasse à Lyon}

Dès la mise en service du nouveau pénétromètre, il a été réalisé des essais comparatifs dans les sols lyonnais dont la géologie est bien connue. Généralement, dans ces sols, la pénétration statique avec un cône électrique ou mécanique est bloquée entre 3 et $4 \mathrm{~m}$ de profondeur. De son côté, la pénétration statique-dynamique lourde ancienne [Sanglerat, 1979] est arrétée soit dans les alluvions compactes ou cimentées, soit, au mieux, en tête de la molasse, vers 20 m de profondeur.

Les premiers essais réalisés avec le nouveau pénétromètre statique-dynamique ont permis d'atteindre, à Lyon, en 1993, une profondeur de $35 \mathrm{~m}$ dont $15 \mathrm{~m}$ dans la molasse, ce qui constitue une amélioration remarquable des performances.

En 1994, sur un autre site lyonnais, le long de l'avenue Foch, les alluvions denses composées de graviers plus ou moins sableux ont été traversées sur $21 \mathrm{~m}$ et ensuite la pénétration a été poursuivie sur $24 \mathrm{~m}$ de profondeur dans la molasse; ce qui constitue un record jamais atteint dans ce type de milieu par aucum autre pénétromètre, qu'il soit dynamique ou statique-dynamique.

Le diagramme de la figure 5 représente les résultats obtenus lors de cette pénétration. Il est bien évident que ce type de reconnaissance, qui donne de précieuses indications sur les caractéristiques de la molasse, est beaucoup moins coûteux que les méthodes usuelles par carottage avec prélèvement d'échantillons et essais de laboratoire.

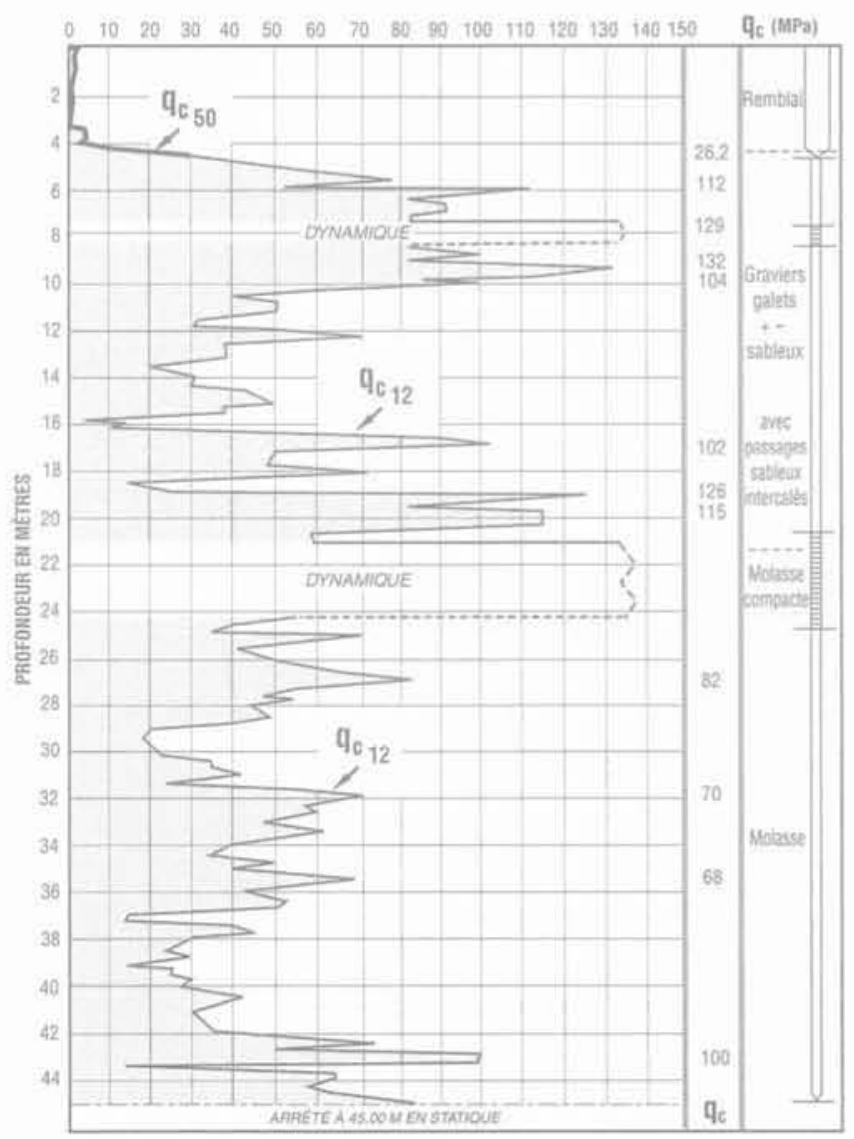

FIG.5 Record de pénétration dans la molasse lyonnaise (avenue Foch à Lyon). Penetration record in dense sandstone.
Pour faciliter la lecture de certains diagrammes, figurent les mentions :

- $q_{-50}$ pour la résistance statique de pointe en $50 \mathrm{~cm}^{2}$; - $\mathrm{q}_{\mathrm{c} 12}$ pour la résistance statique de pointe en $12 \mathrm{~cm}^{2}$.

\section{4}

\section{Essais comparatifs au port autonome du Havre}

Le fonctionnement en statique du cône de $12 \mathrm{~cm}^{2}$ du nouveau pénétromètre est différent de celui du pénétromètre électrique hollandais classique de $10 \mathrm{~cm}^{2}$. Il était donc nécessaire de prouver la validité de la résistance de pointe calculée avec le cône de $12 \mathrm{~cm}^{2}$, en la comparant à la résistance mesurèe tant par le cône électrique de $10 \mathrm{~cm}^{2}$ que par le cône mécanique de $50 \mathrm{~cm}^{2}$ du nouveau pénétromètre.

Des essais comparatifs ont pu être réalisés d'une manière facile car ces trois types de cônes ont pu ètre utilisés pour une étude réelle de fondations au port autonome du Havre en 1993.

Cés essais comparatifs très intéressants ont èté obtenus dans les dépôts sédimentaires du delta de la Seine où l'on trouve des sables fins avec quelques intercalations de tourbe et de petites couches graveleuses.

Ces essais [Sanglerat et al., 1995] ont montré que : a) Sur le plan pratique, les résultats obtenus sont analogues, quel que soit le cône utilisé, électrique de $10 \mathrm{~cm}^{2}$ ou mécanique de $12 \mathrm{~cm}^{2}$ ou de $50 \mathrm{~cm}^{2}$.

b) Il est évident, cependant, que plus le cône est large, plus il a tendance à adoucir les crêtes des diagrammes dues soit à la présence de graviers, soit aux chutes de résistance résultant de couches minces très compressibles.

c) Les résultats obtenus prouvent la validité de la méthode de détermination de $q$, avec le cône de $12 \mathrm{~cm}^{2}$. d) Le cône de $12 \mathrm{~cm}^{2}$, grâce à sa puissance de pénétration. permet d'atteindre en statique des profondeurs beaucoup plus grandes que le cône électrique classique de $10 \mathrm{~cm}^{2}$.

\section{5}

\section{Pont sur l'Arve à Cluses (Haute-Savoie)}

En 1993, un pont à trois travées hyperstatiques de $32 \mathrm{~m}, 19 \mathrm{~m}$ et $32 \mathrm{~m}$, a été construit sur l'Arve lors de la création d'une nouvelle route reliant Marnaz à Thiez. près de Cluses (Haute-Savoie).

A la suite d'une étude de sols basée sur deux essais pressiométriques descendus respectivement à $30,50 \mathrm{~m}$ et $33 \mathrm{~m}$, il avait été décidé d'appuyer les fondations de cet ouvrage d'art important sur des pieux métalliques battus de $25 \mathrm{~m}$ de longueur, travaillant essentiellement au frottement latéral.

Le terrain est constitué par des couches sablo-graveleuses plus ou moins compactes, avec des intercalations de limons et silts, surmontées de remblais superficiels. Sur $10 \mathrm{~m}$ d'épaisseur, existent des lentilles de sols organiques et tourbe.

Un artésianisme important avait été détecté entre $20 \mathrm{~m}$ et $28 \mathrm{~m}$ de profondeur, dans des couches sablograveleuses moyennement compactes. 
En cours de chantier, il est apparu que les pieux s'enfonçaient pratiquement sous leur propre poids, les essais de chargement ont montré que la force portante prévue n'était pas atteinte. $\mathrm{H}$ a donc été décidé d'allonger les pieux de $13 \mathrm{~m}$, ce qui n'a pas été suffisant.

Il a donc fallu allonger à nouveau les pieux de $13 \mathrm{~m}$ pour atteindre ainsi une longueur totale de $51 \mathrm{~m}$.

Si les renforcements ainsi apportés aux pieux ont donné satisfaction pour les deux piles en rivière et pour la culée rive droite, par contre, dès la mise en service de l'ouvrage, la culée rive gauche a subi très rapidement des déformations importantes, ce qui a déclenché une expertise judiciaire pour déterminer la cause des désordres et les travaux confortatifs à réaliser.

Pour déterminer les caractéristiques géotechniques des couches traversées par les pieux et des couches sous-jacentes, il a été alors exécuté quatre essais de pénétration statique Amap'sols qui ont atteint aisément les profondeurs 70,75 et $75 \mathrm{~m}$ et même $82 \mathrm{~m}$ [Sanglerat et al., 1995].

A la suite de ces essais, le pont a été fermé à la circulation pendant trois mois, pour permettre d'améliorer la sécurité de la culée rive gauche par les travaux suivants:

- enlèvement des remblais sur une hauteur de $6 \mathrm{~m}$ pour les remplacer par des remblais allégés en polystyrène expansé :

- mise en place de 8 drains de décharge à débit contrôlé dans la nappe artésienne.

Naturellement, ces travaux confortatifs ont été exẻcutés sous la direction d'un maître d'ceuvre assisté par un géotechnicien.

6.

\section{Pénétration dynamique}

On sait depuis longtemps qu'il faut absolument proscrire la pénétration dynamique en milieu cohérent saturé [Sanglerat, 1979; Sangierat et al., 1984].

Dans les cas où ce type de pénétration est admissible, l'un des problèmes majeur et délicat est la transposition des mesures faites en valeur de résistance dynamique conventionnelle en utilisant, par exemple, la formule des Hollandais. Par ailleurs, l'interprétation de ces résistances dynamiques conventionnelles constitue un sujet qui peut prèter à discussions et à controverses.

Tout problème à ce propos est évité avec le nouveau pénétromètre statique-dynamique.

En effet, grâce à sa puissance, la pénétration statique avec le cône de $12 \mathrm{~cm}^{2}$ peut atteindre 140 à $146 \mathrm{MPa}$ et mème $148 \mathrm{MPa}$ (cf. \$ 7). De ce fait, la pénétration dynamique n'est jamais utilisée que pour traverser des couches extrêmement résistantes. Les contrôles statiques effectués systématiquement permettent d'ailleurs de reprendre la pénétration statique dès que celle-ci chute au-dessous de 130 à $140 \mathrm{MPa}$.

En conséquence, lorsque l'on réalise de la pénétration dynamique, on sait que la résistance statique est toujours très élevée et supérieure en général à 130 ou $140 \mathrm{MPa}$; il n'y a donc plus lieu de se préoccuper de la résistance dynamique conventionnelle, ni de sa transposition en contraintes admissibles. Ceci constitue un avantage important du nouveau pénétromètre statiquedynamique,

\section{7}

\section{Pénétration statique en milieux pulvérulents très denses}

L'un des reproches qui est couramment fait à la pénétration, surtout à la pénétration statique et quelquefois même à la pénétration dynamique, est que ces essais in situ sont bloqués sur des couches très compactes et que l'on ne peut savoir ce qui se passe au-dessous de ces horizons résistants.

Effectivement, ce reproche est souvent justifié, mais cet inconvénient n'existe plus avec le nouveau pénétromètre statique-dynamique.

Nous avons montré, dans les pages qui précèdent. que cet appareil permet de pénétrer, sur de grancles profondeurs, la molasse lyonnaise, pourtant très compacté et présentant souvent une cohésion non négligeable. Pour prouver l'efficacité de la pénétration stafique dans les milieux pulvérulents très denses, nous ne pouvons mieux faire que présenter, à titre d'exemples, six chantiers de la région Rhône-Alpes.

- Sassenage (Isère) (Fig. 6)

- Lyon-La Guillotière (Fig. 7)

- Golf de Villette d'Anthon (Isère) (Fig. 8)

- Pont autoroutier à Jarrie (Isère) (Fig. 9)

- Échirolles (Isère) (Fig. 10)

- Lyon-Porte Dauphine (Fig. 11)

Les valeurs très élevées de $q_{\text {pra }}$ mises en évidence par ces différents diagrammes, peuvent couramment être rencontrées dans les sols sablo-graveleux, très compacts.

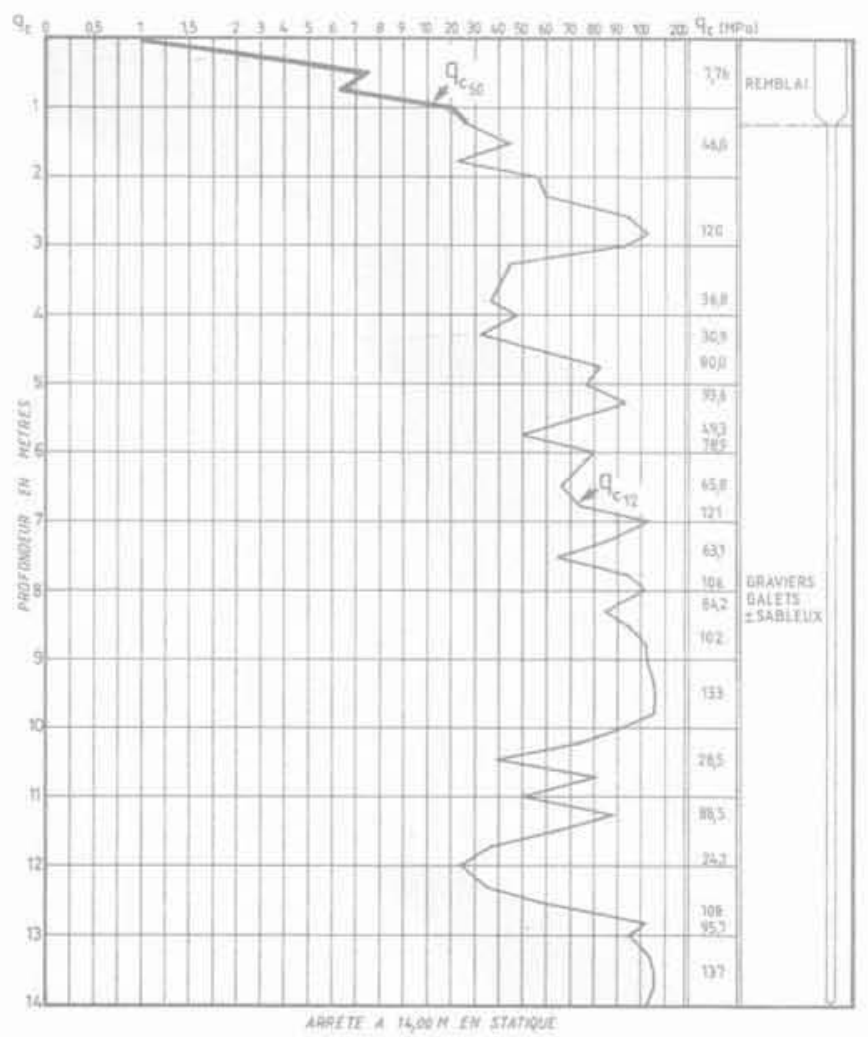

FiG. 6 Pénétration statique dans un gravier sableux très dense à Sassenage (Isère).

Static penetration in very dense sandy gravel in Sassenage (isère). 


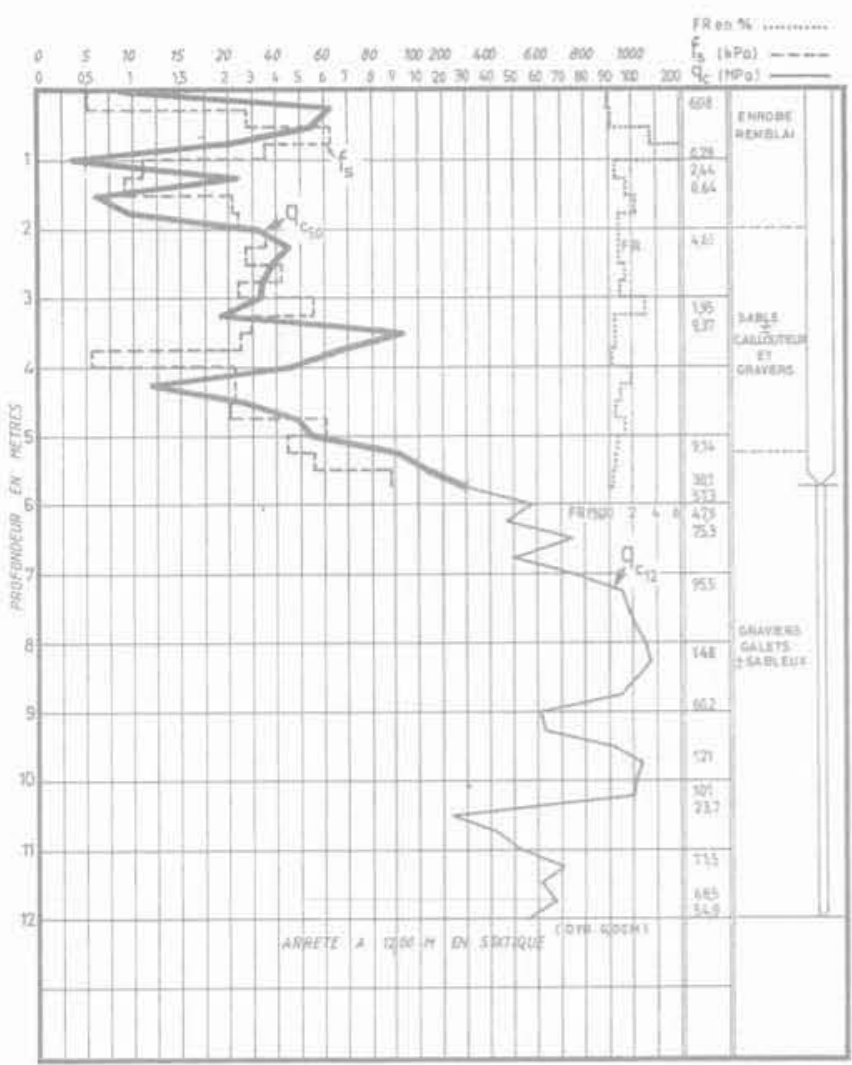

FIG.7 Pénétration statique dans un gravier sableux très dense, ZAC de la Porte-de-laGuillotière à Lyon (Rhône).

Penetration in very dense sandy gravel with cobbles in Lyon (Phòne)

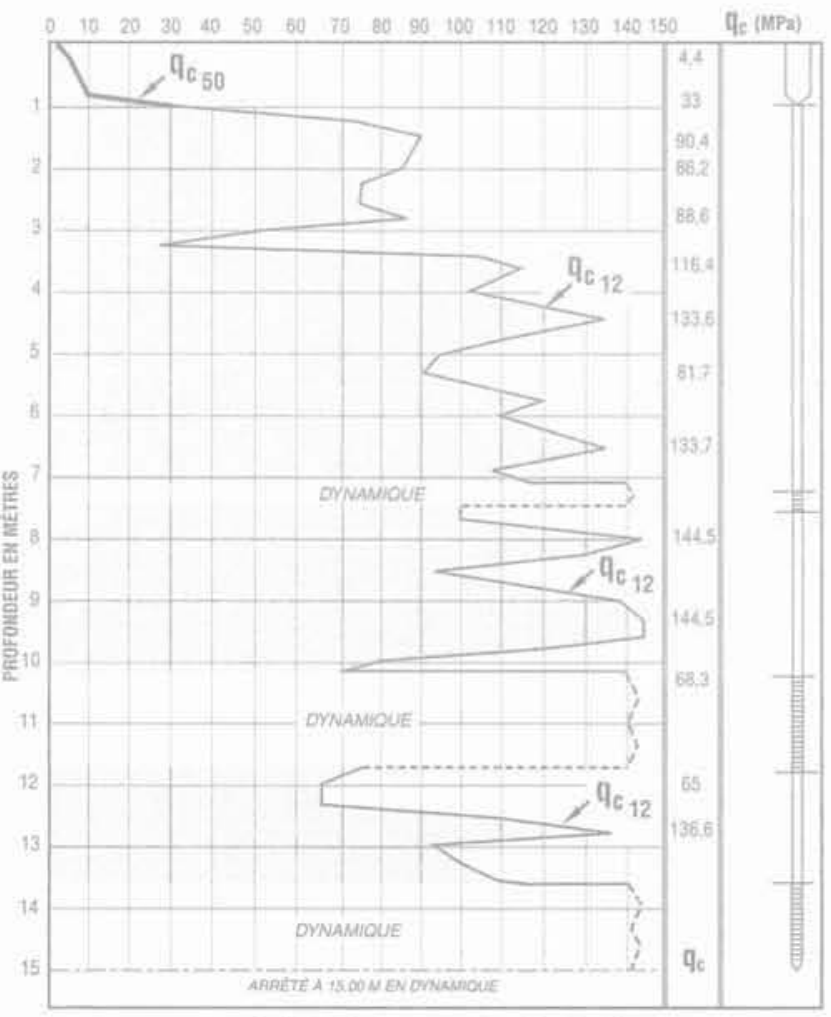

Figure 8 - Pénetration dans un graver asblowa trẹ donoe

FIG. 8 Pénétration dans un gravier sableux très dense à Villette-d'Anthon (Isère).

Penetration in very dense sandy gravel in Villette-d'Anthon (Isere).

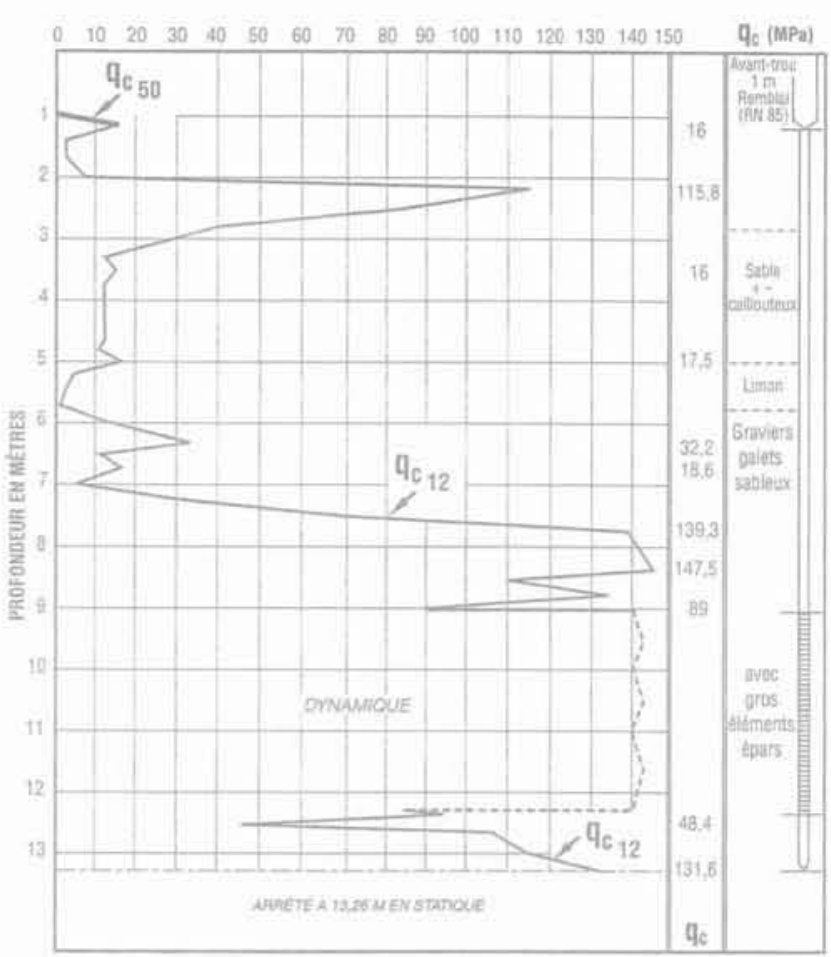

Figure 9 - Pénétration dans un gravier sabloux trés dented

Fig.9 Pénétration dans un gravier sableux très dense, pont autoroutier à Jarrie (lsère). Penetration in very dense sandy gravel with cobbles in Jarrie (Isere).

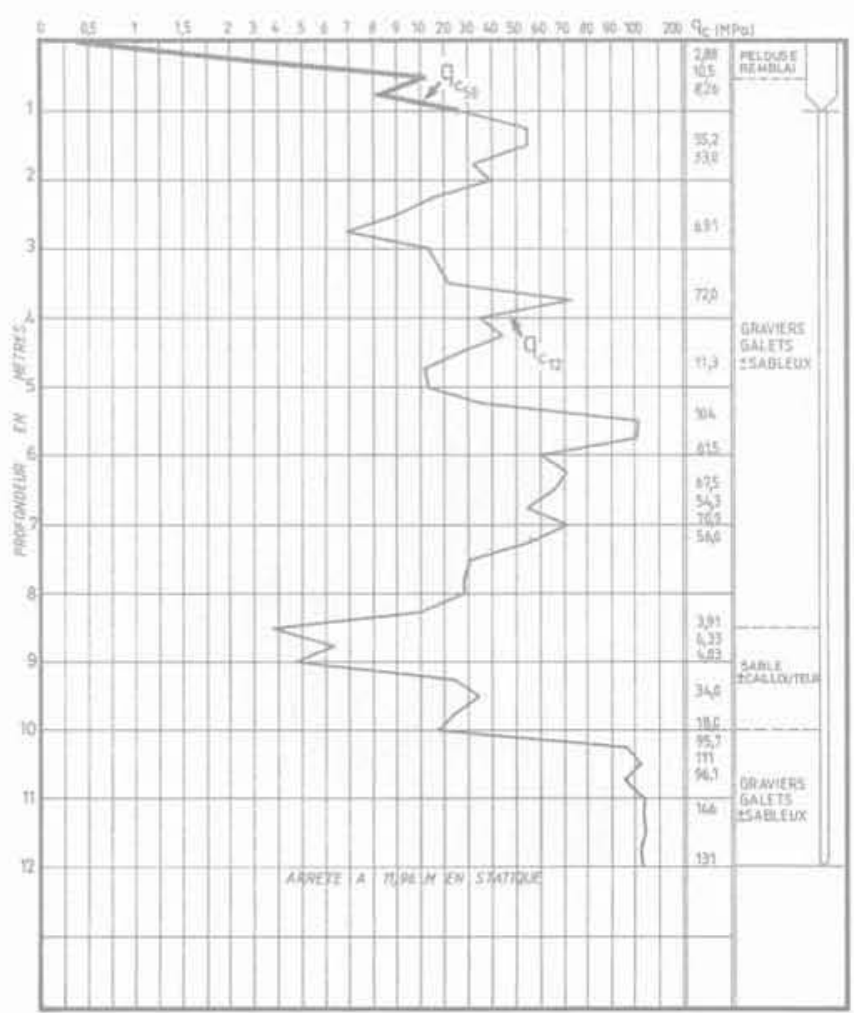

F1G.10 Pénétration statique en milieu pulvérulent très dense ả Échirolles (Isère).

Penetration in very dense sandy gravel with cobbles in Échirolles (Isère) 


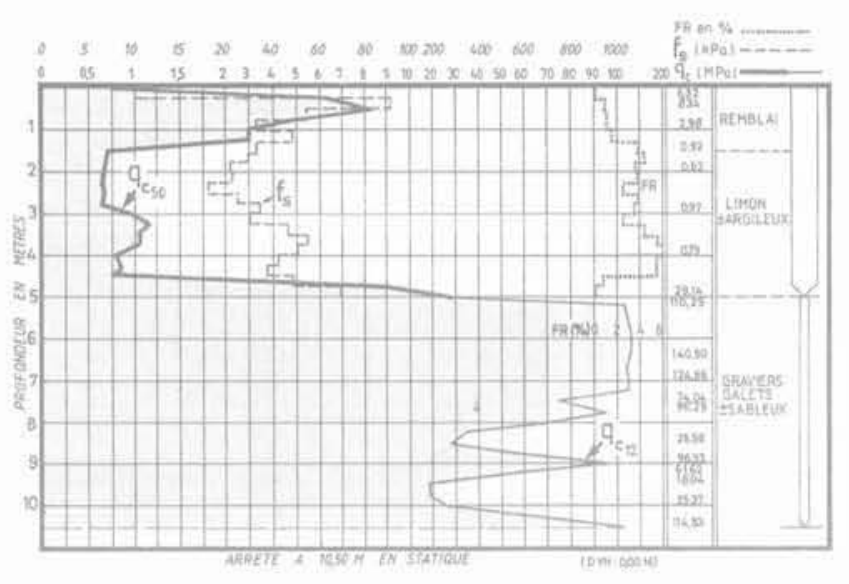

FG. 11 Pénétration dans un gravier sableux très dense, porte Dauphine à Lyon.

Static penetration in very dense gravel and sandy cobbles in Lyon.

\section{Cas des milieux cohérents compacts}

La pénétration statique n'a jamais posé aucun problème pour reconnaitre les sols cohérents compressibles (argile molle, vase, tourbe, limon, silt). C'est même le meilleur essai in situ pour ces types de sol, en particulier lorsqu'ils sont sous la nappe phréatique.

Par contre, le grand reproche que l'on pouvait faire jusqu'en 1992 aux pénétromètres statiques, c'était d'être bloqués sur des couches résistantes, même minces, qui pouvaient sumonter des zones beaucoup plus faibles. Cela constituait un handicap indéniable.

Par ailleurs, jusqu'à présent, tous les pénétromètres statiques rencontraient le refus sur le toit des sols cohérents compacts soit en raison d'une résistance de pointe élevée, soit, le plus souvent, en raison du frottement latéral total cumulé très important.

Si dans le cas des milieux pulvérulents, la pénétration dynamique peut être nécessaire pour traverser des couches indurées ou comportant de gros éléments, par contre il faut reconnaître que dans les milieux cohérents très compacts (type marne surconsolidée), particulièrement sous la nappe phréatique, la pénétration dynamique ne permet pas de progresser d'une manière significative par rapport à la pénétration statique usuelle. Donc, seule la puissance du pénétromètre er statique permet de reconnaître ces types de sol. Or, force est de constater que, jusqu'à présent, tous les pénétromètres étaient bloqués sur le toit des marnes compactes. Par contre, le nouveau pénétromètre statique, compte tenu de sa puissance et de sa conception. fonctionnant tout d'abord avec une pointe de grand diamètre, puis ensuite avec une pointe de petit diamètre, permet de s'affranchir en très grande partie de l'effet néfaste du frottement latéral cumulé et permet de poursuivre la pénétration sur de plus grandes profondeurs.

Citons un exemple récent survenu dans la récion méditerranéenne. On se trouve dans un port où les Grecs et les Romains ont exercé une activité commerciale intense et où subsistent des vestiges archéologiques intéressants.
Le sol est constitué de la façon suivante: -0 à $2 \mathrm{~m}$ : remblais divers :

- de 2 à $8 \mathrm{~m}$ : alluvions modernes de très mauvaise qualité, pouvant comporter des vases et des tourbes: - au-dessous, marnes du Stampien compactes dont la partie supérieure est altérée ou très altérée.

Pour réaliser, dans un tissu urbain fort dense, un parking de $48 \mathrm{~m} \times 77 \mathrm{~m}$, profond de $17 \mathrm{~m}$ environ, il a été prévu une paroi moulée de $60 \mathrm{~cm}$ d'épaisseur, avec trois lits d'ancrage précontraints en partie courante (Fig. 12) et des bracons horizontaux à 45 degrés dans les angles.

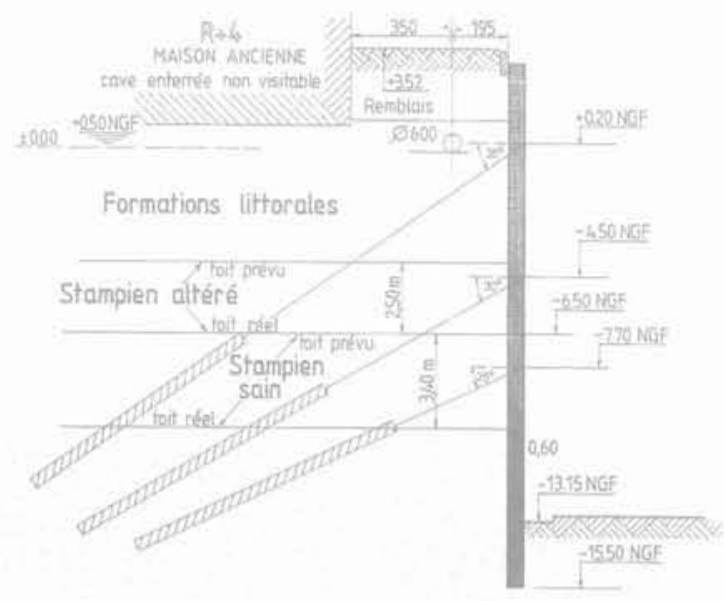

FG. 12 Coupe de la paroi avec trois lits d'ancrages précontraints,

Cross-section of concrete slurry wall showing 3 levels of pre-stressed anchors.

Deux immeubles importants, en maconnerie de pierre du Xvie siècle, se trouvaient à proximité de la fouille, côté est et côté nord.

L'étude géotechnique initiale comportait très peu de sondages profonds mais il était spécifié dans les pièces écrites du marché des entreprises, que le site était bien connu cräce à de nombreuses reconnaissances dans le cadre du creusement de plusieurs tunnels à proximité. De ce fait, les caractéristiques qéotechniques des clifférentes couches existant sur le site étaient dèfinies dans le CCTP par un tableau, tel que le tableau I reproduit ci-après.

Lorsque la fouille a été excavée, la paroi moulée s'est déformée beaucoup plus qué prévu, elle s'est même fissurée à certains endroits, et des tassements importants, de $30 \mathrm{~mm}$ à $60 \mathrm{~mm}$ sont survenus à proximité de l'angle nord-est du parking, entrainant des désordres graves dans l'un des bátiments anciens en maçonnerie de pierres très proches.

Après le sinistre, des essais in situ ont été réalisés, notamment avec le pressiomètre Ménard et avec deux types de pénétromètres statiques : un pénétrométre mécanique et un pénétromètre électrique.

Le pressiomètre n'a pas permis de déterminer avec précision ni le toit exact du Stampien altéré, ni celui du Stampien sain, et les deux pénétromètres n'ont pas pu atteindre le Stampien sain car ils ont été bloqués entre $8 \mathrm{~m}$ et $12 \mathrm{~m}$ de profondeur dans les premiers décimètres du Stampien altéré. 
Or, il fallait reconnaitre exactement, dans la zone des tassements les plus importants et des déformations anormales de la paroi, quelles étaient les épaisseurs exactes des couches de terrain qui exerçaient des poussées sur la paroi litigieuse.

On a donc demandé la réalisation de quatre essais avec le nouveau pénétrométre statique qui ont atteint sans difficulté les profondeurs de $15,75 \mathrm{~m}, 20,02 \mathrm{~m}$. $20,18 \mathrm{~m}$ et $21,14 \mathrm{~m}$, en traversant sur plusieurs mètres non seulement le Stampien altéré, mais également le Stampien sain.

Le diagramme de la figure 13 est fort explicite. II montre que, jusqu'à $10 \mathrm{~m}$ de profondeur, on est dans les alluvions modemes de très médiocre qualité, fort compressibles, donc que le toit du stampien altéré est beaucoup plus bas que prévu puisqu'il était annoncé à 7,50 $\mathrm{m}$ de profondeur.

Par ailleurs, entre $10 \mathrm{~m}$ et $13,50 \mathrm{~m}$, on constate que la résistance de pointe $q_{-50}$ et le frottement latéral $f_{\text {, }}$ augmentent régulièrement, mais sans être très élevés. On se trouve donc dans le Stampien altéré. Enfin, le Stampien sain, qui était annoncé à $10 \mathrm{~m}$ de profondeur, se trouve en réalité $13,50 \mathrm{~m}$ de profondeur.

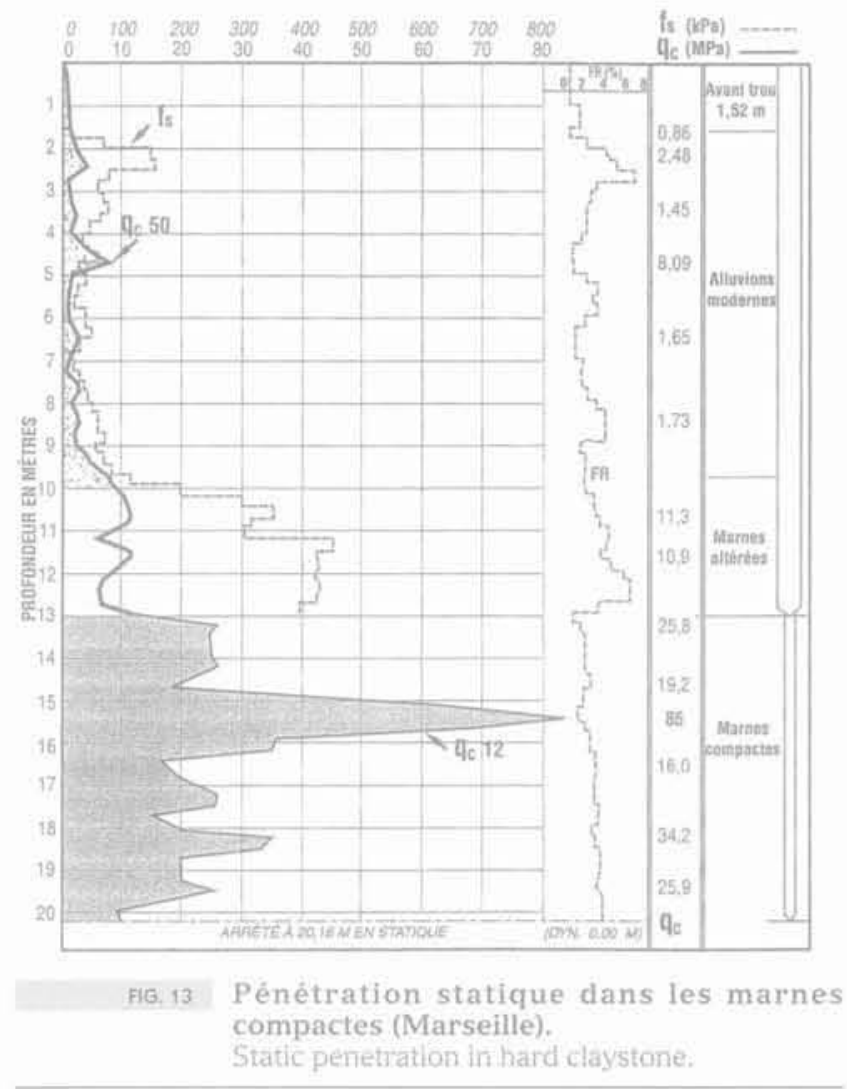

Le tableau II ci-dessous donne les caractéristiques pénétrométriques de ces couches.

La figure 12 donne une coupe nord-sud du chantier avec les toits prévus et réels du stampien altéré, ainsi que les toits prévus et réels du stampien sain.

Naturellement, lorsqu'il y a des erreurs de niveau aussi importantes, les poussées sont beaucoup plus grandes que prévu. Il n'est donc pas étonnant que les parois aient été fortement déformées et que cela ait entraîné des tassements importants dans les zones adjacentes très compressibles, d'où des tassements différentiels importants ayant provoqué des désordres graves dans des maçonneries de pierre fort vétustes (XVI siècle).

TABLEAUil Caractéristiques pénétrométriques.

\begin{tabular}{|c|}
\hline $\begin{array}{l}\text { Stampien altéré } \\
4<\mathrm{q}_{\mathrm{Lso}}<9 \mathrm{MPa} \\
100<\mathrm{f}_{\mathrm{s}}<400 \mathrm{kPa} \\
2<\mathrm{FR}<5 \%\end{array}$ \\
\hline $\begin{array}{c}\text { Stampien sain } \\
10<q_{c t 2}<85 \mathrm{MPa}\end{array}$ \\
\hline
\end{tabular}

\section{9}

\section{Contrôle des remblais sablo-graveleux}

La pénétration statique est un excellent moyen pour contrôler la compacité, donc la qualité, d'un remblai sablo-graveleux, que ce soit à terre ou, a fortiori, sous l'eau.

Rappelons les critères de contrōle que l'expérience nous a permis d'établir il y a de longues années [Sanglerat, 1979].

Relation entre compactage et $\mathrm{q}_{c}$.

\begin{tabular}{c|c|c}
\multicolumn{1}{c|}{ Type } & \multirow{2}{*}{$\mathcal{q}_{c}(\mathrm{MPa})$} \\
\cline { 1 - 2 } Gravier propre & Lẩche & \multirow{2}{*}{1,5 à 2,5} \\
\hline Gravier sableux & Làche & 3 à 4,5 \\
\hline Gravier sableux & Moyennement compact & 8 à 12 \\
\hline Gravier sableux & Très bien compacté & $>20$ \\
\hline
\end{tabular}

TAsLEAUI Caractéristiques géotechniques des différentes couches selon le CCTP.

\begin{tabular}{l|c|c|c|c|c|c|c}
\multirow{2}{*}{ Faciès } & \multicolumn{3}{|c|}{ Densité } & $K$ & \multicolumn{2}{c|}{ Court terme } & \multicolumn{2}{c}{ Long terme } \\
\cline { 2 - 8 } & $\gamma$ & $\gamma^{\prime}$ & $\mathrm{kPa} / \mathrm{m}$ & $\varphi^{\circ}$ & $\mathrm{c}(\mathrm{kPa})$ & $\varphi^{\prime}$ & $\mathrm{c}^{\prime}(\mathrm{kPa})$ \\
\hline Remblais & 2 & 1 & 5000 & 30 & 0 & 30 & 0 \\
\hline Formations littorales & 1,8 & 0,8 & 5.000 & 25 & 15 & 30 & 25 \\
\hline Stampien altéré & 2,1 & 1,1 & 25000 & 20 & 80 & 250 \\
\hline Stampien sain & 2,1 & 1,1 & 50000 & 25 & 200 & 30 & 130 \\
\hline
\end{tabular}


Naturellement, lorsque les remblais sablo-graveleux sont très denses, le pénétromètre électrique ne peut être utilisé, car sa pointe risquerait d'être endommagée. Par contre, le nouveau pénétromètre statiquemécanique est très efficace.

Nous avons eu l'occasion de l'utiliser à plusieurs reprises, comme plusieurs de nos confrères, lors d'expertises judiciaires, pour déterminer notamment l'épaisseur et la qualité de remblais sablo-graveleux sous dallage de béton (entrepôts, usines, etc.). Il s'agissait en général de rechercher à qui pouvaient être imputés les tassements différentiels survenus dans les dallages.

Les mesures $q$ enregistrées tous les $2 \mathrm{~cm}$ permettent de tracer des diagrammes tels que ceux reproduits sur la figure 14. Cette figure montre immédiatement que si dans la couche supérieure du remblai la compacité est très bonne $\left(q_{f}>20 \mathrm{MPa}\right)$, par contre ce n'est plus le cas en partie inférjeure. On constate également que l'épaisseur du gravier est fort irrégulière selon les emplacements.

Il est bien évident qu'aucun autre type d'essais n'est capable de fournir une telle précision, ni pour les épaisseurs, ni pour les variations de compacité à différentes profondeurs.

\section{0}

\section{Contrôle des colonnes ballastées}

La réglementation française (DTU 13.2 Fondations profondes) stipule que les colonnes ballastées peuvent être réceptionnées grâce au pénétromètre statique, dès lors que :

$$
\mathrm{q}_{\mathrm{c}}>10 \mathrm{MPa} \text {. }
$$

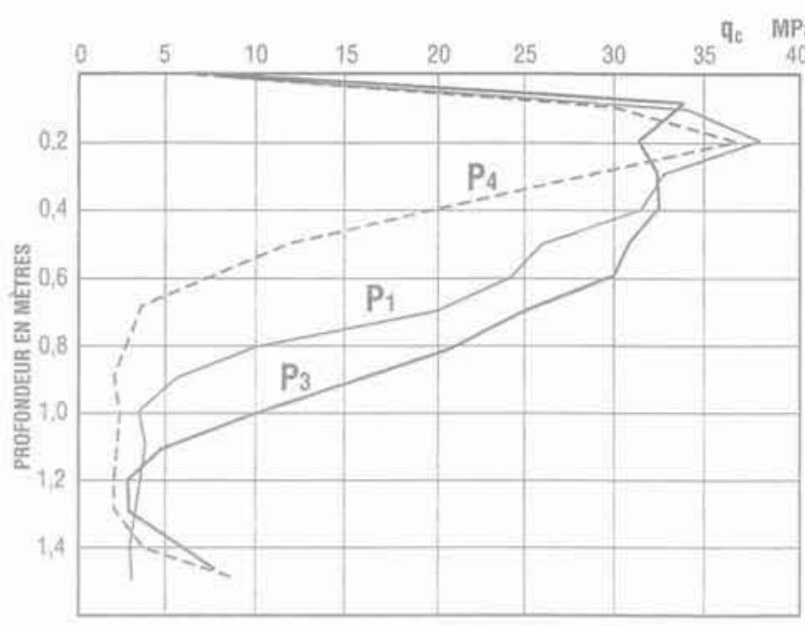

FG.14 Contrôle de la compacité d'un remblai sablo-graveleux sous dallage béton (Verdun-sur-le-Doubs).

Fill density check under concrete floor.

Mais trop souvent, les cônes de petite section (10 à $15 \mathrm{~cm}^{2}$ ) des pénétromètres usuels sont déviés, par certains graviers, hors de la colonne elle-même, Ainsi q. chute car la pointe pénètre alors dans le sol naturel adjacent non densifié.

Les mesures obtenues sont donc sans signification ; elles peuvent même conduire à des conclusions erronées (colonne jugée mal densifiée),

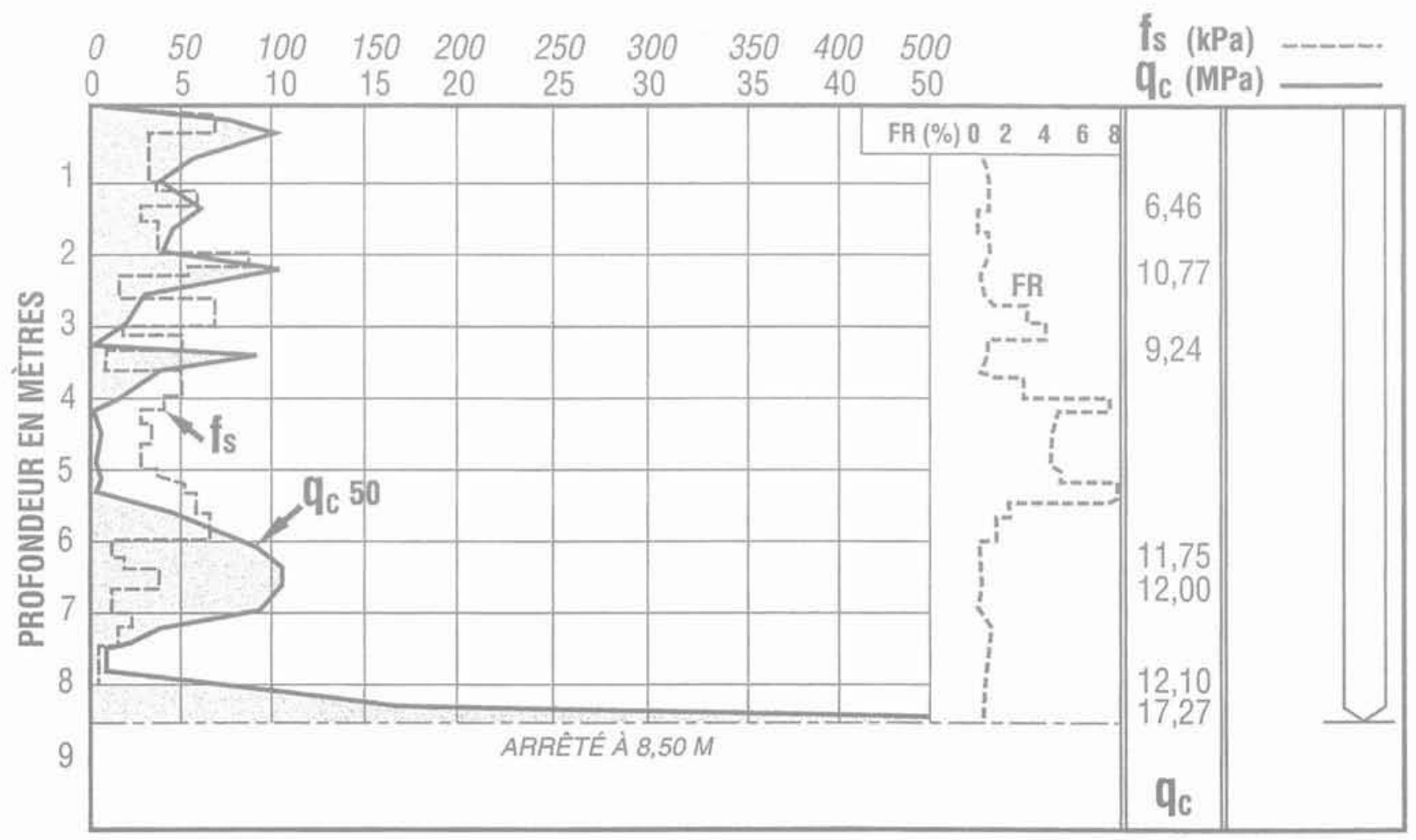




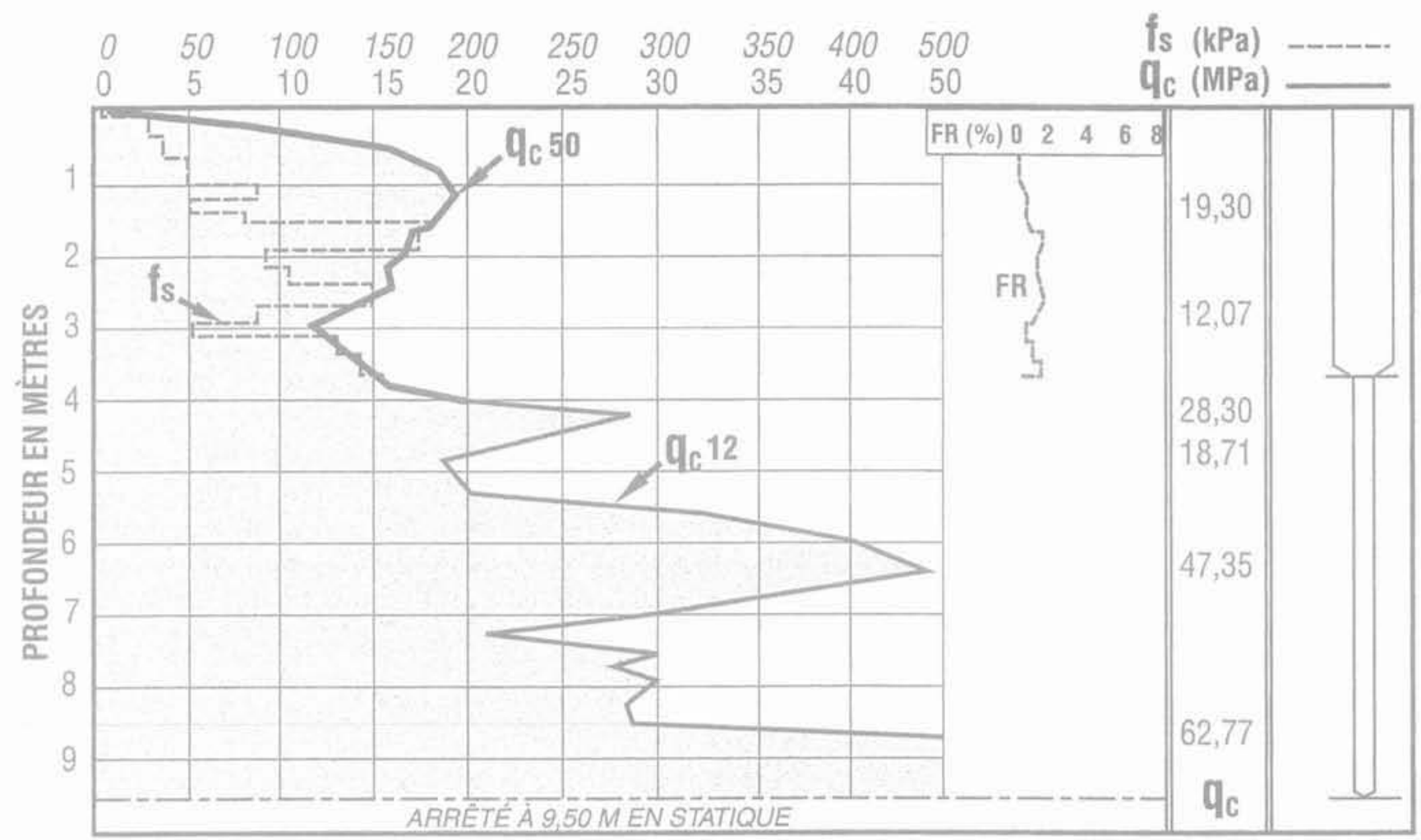

FG. 158 Pénétration statique dans colonne ballastée (région parisienne),

Static penetration in densifjed stone column.

Mais le nouveau pénétromètre avec sa grande pointe de $50 \mathrm{~cm}^{2}$, gràce à sa grande rigidité et à sá grande puissance, est capable de traverser la totalité des colonnes ballastées sans ètre dévié, ni bloqué par un gravier de grandes dimensions. On peut citer par exemple un résultat obtenu récemment en région parisienne (Fig. 15). La colonne testée est continue et d'excellente qualité :

$$
12<q_{c}<47 \mathrm{MPa}
$$

Le nouveau pénétromètre statique permet de distinguer rapidement et sûrement les colonnes mal densifiées qui doivent être reprises, et les colonnes bien densifiées qui peuvent ètre réceptionnées.

\section{1}

\section{Coût des essais}

L'expérience pratique de ces dernières années en France a montré que les reconnaissances faites avec le pénétromètre statique-dynamique étaient d'une manière générale beaucoup moins coûteusés que les autres méthodes souvent utilisées, tout en fournissant beaucoup plus de renseignements, puisque les mesures sont faites en continu et enregistrées tous les deux centimètres. Nous avons constaté que le prix par mètre de pénétration d'une reconnaissance est en général :

- moitié moins élevé que celui d'une reconnaissance avec des essais pressiométriques exécutés selon les règles de l'art:

- de 30 à $33 \%$ du coût d'un sondage carotté avec prise d'échantillons et enregistrement de paramètres.

\section{2}

\section{Pénétration dans les graviers hollandais}

A t'occasion du $12^{\mathrm{c}}$ Congrès européen de Mécanique des Sols à Amsterdam, du 7 au 10 juin 1999, le pénétromètre AMAP'sols a été présenté aux participants. De très nombreux géotechniciens ont été intéressés par ses possibilités.

En cette même période, la Société Van dèn Berg de Heerenveen ètait confrontée à un problème difficile, dans l'Est de la Hollande, à Nijmegen. Cette ville, que les Français appellent Nimègue, est située près de la frontière allemande, à proximité du Rhin.

Pour éviter tous les désagréments dus à la neige, au givre et aux conséquences catastrophiques du dégel, le grand club de football NEC a décidé d"installer des tubes de chauffage encastrés horizontalement à $25 \mathrm{~cm}$ sous sa pelouse, les apports calorifiques provenant d'échangeurs de chaleur foncés verticalement à grande profondeur, et d'une pompe à chaleur. Naturellement, en été, le mème système pourrait être utilisé pour «climatiser n les bâtiments ou simplement pour restituer des calories dans le sol afin de ne pas perturber son équilibre thermique global à long terme (héliogéothermie).

Les avantages de ce système non polluant sont: - diminution d'utilisation de combustible fossile ;

- moins de $\mathrm{CO}_{2}$ dans l'atmosphère ;

- au moins $50 \%$ d'économie sur les frais de chauffage: - moins d'entretien. 
Les couches du terrain étant généralement molles aux Pays-Bas, on utilise le pénétromètre pour mettre en place les échangeurs jusqu'à la profondeur désirée. Pour ce faire, on enfonce tout d'abord des tubes fourreaux métalliques $\emptyset 56 / 40 \mathrm{~mm}$, avec une pointe perdue $\emptyset 64 \mathrm{~mm}$. Dans ces tubes, on introduit les échangeurs en polyéthylène $630 \mathrm{~mm}$. Puis on arrache les tubes fourreaux en laissant les échangeurs en place, ainsi que la pointe perdue.

Mais, à Nimègue, le terrain est constitué de sable de très grande épaisseur, comportant localement, à diverses profondeurs, soit des couches peu résistantes de loess, argile et tourbe, soit des couches de graviers denses, avec des zones indurées. L'épaisseur de ces couches intercalées peut varier de 1 à 3 ou $4 \mathrm{~m}$. Il s'agit des alluvions du Rhin. La nappe phréatique, située vers 9 mètres de profondeur, peut fluctuer de plusieurs mètres.

Il avait été décidé d'utiliser deux pénétromètres statiques hollandais pour foncer ces tubes fourreaux verticalement, mais ceux-ci ont été bloqués, en certaines zones, à 16 metres de profondeur environ sur une couche de graviers compacts du Rhin. Or, il fallait descendre impérativement à au moins 28 mètres pour avoir un écart de température suffisant pour l'eau des pompes à chaleur.

Van den Berg, qui a construit en grande partie le nouveau pénétromètre, en 1992, sachant que ce dernier était plus puissant que tous les autres pénétromètres statiques existants, a proposé d'essayer le pénétromètre français pour résoudre son problème.

Non seulement le nouveau pénétromètre a permis d'atteindre la profondeur prescrite de 28 mètres, mais il a pu, en outre, enfoncer sans difficulté, alternativement en statique et en dynamique quand cela était nécessaire, les tubes fourreaux $\varnothing 56 / 40 \mathrm{~mm}$ jusqu'à 40 mètres de profondeur. Cela a constitué un grand succès quí, en outre, est plus favorable pour la température de $l^{\prime}$ eau recherchée $\left(11^{\circ}\right.$ à $28 \mathrm{~m}$ et $12^{\circ}$ à $\left.40 \mathrm{~m}\right)$. La pointe perdue de $\varnothing 64 \mathrm{~mm}$ est pourvue d'un clips qui retient le polyéthylène $\varnothing 30 \mathrm{~mm}$ lors de la remontée du tube $\varnothing 56 / 40 \mathrm{~mm}$.

C'est ainsi qu'en juin 1999, le nouveau pénétromètre a pu enfoncer, dans des délais très courts, 44 tubes de 40 mètres dans les graviers compacts du Rhin qui avaient souvent bloqué, vers 16 mètres, les autres appareils.

Le succès de ce pénétromètre, dans le pays qui a vu la naissance de la pénétration et son large développement, méritait d'être signalé aux lecteurs de la RFG, car il confirme les résultats exceptionnels obtenus par cet appareil dans les graviers du Rhóne et autres rivières des Alpes.

Précisons qu'un essai de pénétration statique mécanique a été réalisé pour déterminer les caractéristiques des différentes couches alluvionnaires du Rhin. Il est représenté sur la figure 16 . Il met en évidence les faits suivants :

1. la pointe mécanique $\varnothing 80 \mathrm{~mm}$, donnant $\mathrm{q}_{\text {an a été }}$ bloquée à $15,50 \mathrm{~m}$ sur le toit des graviers denses du Rhin qui avait arrêté initialement les tubes fourreaux sensiblement à cette profondeur;

2. la pointe mécanique $\varnothing 39 \mathrm{~mm}$, donnant $\mathrm{q}_{-12}$, a atteint sans difficulté, en statique, $40 \mathrm{~m}$ de profondeur.

Les graviers du Rhin, entre $15,50 \mathrm{~m}$ et $18,50 \mathrm{~m}$, ont pour caractéristiques pénétrométriques:

$$
18<q_{c+2}<46 \mathrm{MPa}
$$

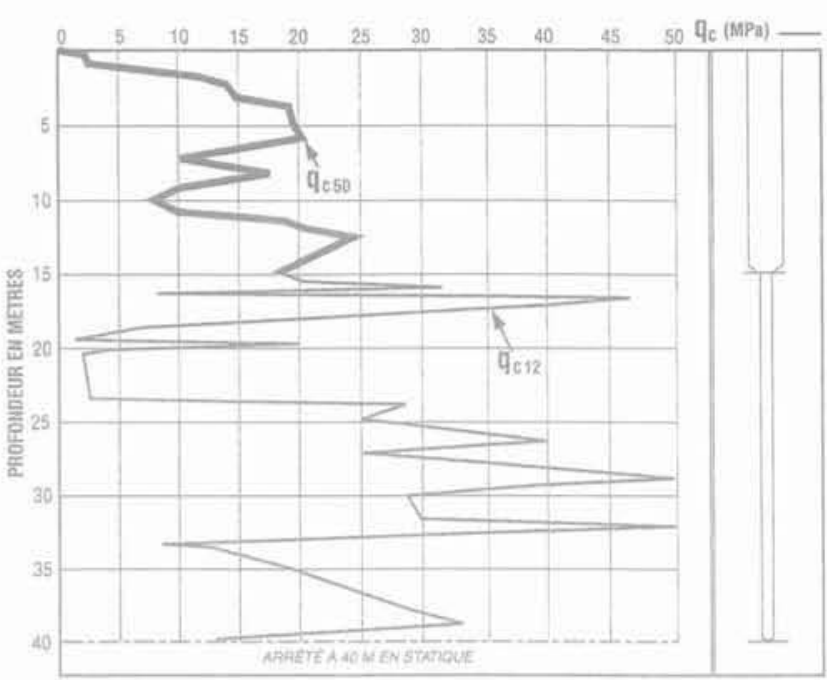

Fig. 16 Pénétration statique dans les graviers sableux denses du Rhin à Nimègue.

Static penetration in very dense sandy gravel ir Nijmegen,

II n'est donc pas étonnant que le fonçage initial des fourreaux provisoires des échangeurs de chaleur, équipés d'une pointe perdue $\varnothing 64 \mathrm{~mm}$, n'ait pu, compte tenu de l'effort de pointe et du frottement latéral total. être poursuivi jusqu'à 40 m de profondeur par les pénétromètres locaux.

Cette expérience de Nimègue montre que ces derniers n'ont pas été utilisés dans leur domaine d'application classique où ils conservent toutes leurs qualités.

\section{Remarque :}

Dans le cas d'un terrain pollué en surface, si l'on doit recourir au forage d'un avant-trou pour la mise en place d'échangeurs de chaleur, il faut, afin d'éviter la transmission de la pollution aux couches profondes. utiliser des techniques spéciales qui sont bien connues aujourd'hui mais onéreuses.

Naturellement, foncer alors des tubes fourreatux équipés d'une pointe perdue, à l'aide d'un pénétromètre, constitue une méthode sure et beaucoup plus économique.

\section{3}

\section{Conclusion}

Les résultats présentés ci-dessus montrent que trois importantes conclusions peuvent être tirées.

1) Le nouveau pénétromètre statique-dynamique permet d'atteindre des pénétrations record dans les sols denses ou compacts, qu'ils soient pulvérulents ou cohérents.

2) Toutes les variations de résistance sont très bien mises en évidence, aussi bien dans les sols denses ou moyennement denses que dans les sols très compressibles.

3) Une reconnaissance à l'aide du nouveau pénétromètre statique-dynamique correspond au meilleur rapport qualité/prix

Il est évident que ce nouveau pénétromètre statique-dynamique permet, par la large gamme des 
pointes pouvant être utilisées, de reconnaitre des sols, même très résistants, d'une manière efficace et rapide. Ses déplacements et sa mise en place sont grandement facilités par le chenillard et ses vérins (Fig. 2).

Par ailleurs, il met à la disposition de l'utilisateur le piézocône et l'envirocône qui permettent de recueillir des données complémentaires extrêmement utiles dans certains cas particuliers et notamment dans la lutte antipollution, ainsi que dans la détermination des terrains liquéfiables sous l'action des séismes [Robertson, 1995; Robertson et al., 1998].

Nul doute que cet appareil ne soit appelé à un large développement, non seulement en France mais également dans de nombreux autres pays, car il constitue, à notre avis, un grand progrès de la technique de pénétration en 1999 .

\section{Bibliographie}

[1] Begemann H.D.S. - The friction lacket cone as an aid in determining the soil profile 3, 6th CFMSF, Montreal, vol. 1. 1975, p. $17-20$

[2] Schmertmann J.H. - - Static cone penetrometer for soll exploration $\mathrm{n}$. Civit Engineering, vol. 37, $n^{\circ} 6,1967$, p. 71-73,

[3] Mitchell J.K., Brandon T.1. - "Analysis and use of CPT in earth quake and environnement engineering x. ISC'98, 1998, p. 69-97.

[4] Sanglerat G. - « It took 2000 years of penetration testing to arrive at the AMAP'sols static-dynamic penetrome- ter w. Symposium on Development in Geotechnical Engineering, Bangkok, 1994. p. 101-105

[5] Sanglerat G, Petit-Maire M., Bardot F., Savasta P. - u Additional test results of the AMAP'sols static dynamic penetrometer v, CPT'95, Linkôping (Sweden), 1995, vol. 2. p. 85-91.

[6] Sanglerat G. - The penetrometer and soil exploration. Second enlarged edition, Amsterdam, New York, Elsevier, $1979,448 \mathrm{p}$.

[7] Sanglerat G., Olivari G., Cambou B. - Practical Problems in SMFE. Vol. I. Physical characteristics of soils, plasticity, settlement calculations, interpretation of in situ tests. Amsterdam, Elsevier, 1984, 283 p.

[8] Robertson P.K. $-u$. Application of CPT to evaluate liquefaction potential \$. CPT 95 . Linkôping (Sweden), vol. 3, 1995, p. 57 79, Swedish Geotechnical Society.

[9] Lunne I, Robertson P.K., Powell J.J.M. - CPT in geotechnical practice, New York, Blackie Academic, 1998, 312 p.

[10] Robertson P.K., Wride C.E. - $\pi$ Evaluation cyclic liquefaction potential using the CPT 1. Canadian Geotech, J35, 1998, p. $442-459$ 